\title{
QUASI ADDITIVE SET FUNCTIONS AND THE CONCEPT OF INTEGRAL OVER A VARIETY
}

\author{
BY \\ LAMBERTO CESARI( ${ }^{(1)}$
}

In the last years a great deal of research has been done on the axiomatization of the concept of linear integral over a variety $T$, but very little for the nonlinear integrals $\Im=\int_{T} f(p, q), p=\left(p_{1}, \cdots, p_{m}\right), q=\left(q_{1}, \cdots, q_{k}\right)$, though they have their natural place in Volterra's functional analysis. In the present paper we give the first elements of a very general axiomatization concerning such integrals. This is done by introducing a concept of quasi normality (quasi additivity, quasi subadditivity, etc.) which extends Banach's concept of normality. As we will see, if $\phi(I)$ is a quasi additive set function of bounded variation, then the integral $\Im(f, T, \phi)$ of a function $f(p, q)$ over any continuous parametric mapping $T$ with respect to $\phi$ ( $f$ positive homogeneous of degree one in $q$ but not necessarily linear) can be defined by a process of limit on the set function $\Phi(I)=f[T(\tau), \phi(I)], \tau \in I$, and this set function is proved to be itself quasi additive [ $\$ 6$, Theorem (i)]. It appears, therefore, that the concept of quasi additivity is a generalization of the usual additivity which is reproduced by the integrand $f$, though the latter is not linear. The integral $\Im$ is obtained by means of a process of limit involving a mesh $\delta$ which is defined axiomatically. Such a process is more general than usual since $\delta$ is not required to decrease nor approach zero by refinements only. Examples of this situation are known ( $\$ 4)$. In successive papers we will discuss the problem of extension and other properties of the integral $\Im$ in connection with measure theory, Radon-Nikodym derivatives, and weak convergence.

The present integral $\Im$ contains, as a particular case, the concept of integral over a continuous surface $T$ of finite area, as defined by the author and used in surface area theory and the calculus of variations by L. Sigalov, J. M. Danskin, V. E. Bononcini, J. Cecconi, L. H. Turner, and the author. In this particular situation $\phi(I)$ is the usual vector signed area of the same mapping $T$.

As is natural, the same integral $\Im$ contains, as a particular case, the classic Weierstrass integral over a curve of finite Jordan length.

Finally, the process of limit considered in the present paper can be thought of as extending Burkill's integration process for normal interval functions. Burkill's integral has been used in connection with total variation, Jordan

Presented to the Society, October 25, 1958 under the title Quasi additive set functions and the concept of integral; received by the editors February 23, 1961.

(1) This research has been supported by ARDC, project AF 18(600)-1484. 
length, and area of nonparametric surfaces by T. Radó, S. Saks, L. Tonelli and others. On this subject we mention the monograph of S. Kempisty [2].

For the bibliography on the general subject see [1]. In particular, for some extensions of the usual requirements in connection with Burkill integration, see, e.g., L. Tonelli [6a] and H. Kober [3].

1. The concept of mesh. We shall denote by $A$ a given set, or space, by $\{I\}$ a collection of subsets $I$ of $A$, by $\mathfrak{D}$ a nonempty family of finite systems $D$ of sets $I \in\{I\}$, i.e., $D=[I]=\left[I_{1}, \cdots, I_{N}\right]$, and $D$ is not necessarily a partition of $A$. We shall denote by $\delta(D), D \in \mathfrak{D}$, a real function defined for all systems $D \in \mathfrak{D}$. We shall denote the sets $I$ as "intervals" and $\delta(D)$ as the "mesh of $D$," or a "mesh function." Concerning the systems $D \in \mathfrak{D}$ we will make either of the following hypotheses:

(b) Either ( $\left.\mathrm{b}^{\prime}\right) A$ is any set, and the sets $I$ of each system $D \in \mathfrak{D}$ are disjoint, i.e., $I, J \in D$ implies $I \cap J=\varnothing, \varnothing$ the empty set; or $\left(\mathrm{b}^{\prime \prime}\right) A$ is a topological space, $\mathfrak{U}$ is the collection of all open sets $U$ of $A$, each set $I \in\{I\}$ possesses interior points, and the sets $I$ of each system $D \in \mathscr{D}$ are nonoverlapping, i.e., $I, J \in D$ implies $I^{0}, J^{0} \neq \varnothing \varnothing, I^{0} \cap J^{0}=I^{*} \cap J^{0}=I^{0} \cap J^{*}=\varnothing$, where ${ }^{0}$ and * denote the subsets of the interior and boundary points of a set in $A$ (in the topology $\mathfrak{U}$ ).

Concerning the mesh function $\delta(D)$ we make the following hypotheses:

$\left(\mathrm{d}_{1}\right) 0<\delta(D)<+\infty$ for every $D \in \mathfrak{D}$;

$\left(\mathrm{d}_{2}\right)$ Given $\epsilon>0$ there are systems $D \in \mathcal{D}$ with $0<\delta(D)<\epsilon$.

Note that $\delta(D)$ is a property of the whole system $D$. It is not requested that any system $D \in \mathfrak{D}$ has in $\mathfrak{D}$ some refinement $D^{\prime}$, and, if this happens, no relation is implied between $\delta(D)$ and $\delta\left(D^{\prime}\right)$.

Let $\phi(I)=\left[\phi_{1}(I), \cdots, \phi_{k}(I)\right], I \in\{I\}$, be any real-valued interval vector function defined for every $I \in\{I\}$. If $k=1$ we say that $\phi(I)$ is an interval scalar function. By $\|\phi\|$ we shall denote the Euclidean norm.

For every $r=1, \cdots, k$, we shall denote by $* \mathfrak{B}_{r}={ }_{*} \mathfrak{B}\left(\phi_{r}, A\right),{ }^{*} \mathfrak{B}_{r}={ }^{*} \mathfrak{B}\left(\phi_{r}, A\right)$ the numbers

$$
\begin{aligned}
& * \mathfrak{B}_{r}=* \mathfrak{B}\left(\phi_{r}, A\right)=\liminf _{\delta(D) \rightarrow 0} \sum_{I \in D} \phi_{r}(I), \\
& * \mathfrak{B}_{r}=* \mathfrak{B}\left(\phi_{r}, A\right)=\limsup _{\delta(D) \rightarrow 0} \sum_{I \in D} \phi_{r}(I),
\end{aligned}
$$

$-\infty \leqq * \mathfrak{B}_{r} \leqq * \mathfrak{B}_{r} \leqq+\infty$, where $D=[I]$ denotes any system $D \in \mathfrak{D}$. The numbers $* \mathfrak{B}_{r},{ }^{*} \mathfrak{B}_{r}$ could be denoted as Burkill-type lower and upper integrals of $\phi_{r}$. Finally, we will put $* \mathfrak{B}=\left({ }_{*} \mathfrak{B}_{1}, \cdots, * \mathfrak{B}_{k}\right),{ }^{*} \mathfrak{B}=\left(* \mathfrak{B}_{1}, \cdots, * \mathfrak{B}_{k}\right)$. If ${ }_{*} \mathfrak{B}_{r}=* \mathfrak{B}_{r}$ (finite, or not), $r=1, \cdots, k$, i.e., if the limit exists

$$
\mathfrak{B}_{r}=\mathfrak{B}\left(\phi_{r}, A\right)=\lim _{\delta(D) \rightarrow 0} \sum_{I \in D} \phi_{r}(I), \quad r=1, \cdots, k,
$$

we shall denote by $\mathfrak{B}=\mathfrak{B}(\phi, A)$ the vector (or Burkill-type integral) 


$$
\mathfrak{B}=\mathfrak{B}(\phi, A)=\lim _{\delta(D) \rightarrow 0} \sum_{I \in D} \phi(I) .
$$

For nonnegative scalar functions $\psi$ we will find it convenient to denote $* \mathfrak{B}(\psi)$, ${ }^{*} \mathfrak{B}(\psi), \mathfrak{B}(\psi)$ by $* V(\psi),{ }^{*} V(\psi), V(\psi)$ respectively. Thus $0 \leqq * V \leqq * V \leqq+\infty$, and $0 \leqq V \leqq+\infty$ if $V$ exists. It may occur that $\phi(I), \psi(I)$ depend also on arbitrary parameters, and that the lim inf, lim sup, above are taken for all possible values of the parameters. In this case the limits above, if they exist are then independent of the choice of these parameters.

2. Quasi normal functions. Let $\phi(I)=\left(\phi_{1}, \cdots, \phi_{k}\right), I \in\{I\}$, be any realvalued interval vector function. We shall say that $\phi(I)$ is quasi additive (with respect to the mesh function $\delta(D)$ and the family $D$ ) provided

$(\phi)$ given $\epsilon>0$ there is a number $\eta=\eta(\epsilon)$ such that, if $D_{0}=[I] \in \mathfrak{D}$ is any system with $\delta\left(D_{0}\right)<\eta$, then there is also a number $\lambda=\lambda\left(\epsilon, D_{0}\right)>0$ such that, for every system $D=[J] \in \mathfrak{D}$ with $\delta(D)<\lambda$ we have

$$
\begin{array}{r}
\sum_{I \in D_{0}}\left\|\sum_{J \in I} \phi(J)-\phi(I)\right\|<\epsilon, \\
\sum_{J \Phi I}\|\phi(J)\|<\epsilon,
\end{array}
$$

where the last sum is taken for all $J \in D, J \nsubseteq I$ for any $I \in D_{0}$.

If $m$ is any real number, let $m^{+}, m^{-}$be, as usual, the numbers $m^{+}$ $=(|m|+m) / 2, m^{-}=(|m|-m) / 2$.

An interval scalar function $\psi(I)$ is said to be quasi subadditive (with respect to the mesh function $\delta(D)$ and the family $D$ ) provided the statement $(\psi)$ holds which is analogous to $(\phi)$ where $\left(\phi_{1}\right),\left(\phi_{2}\right)$ are replaced by the single relation

$$
\sum_{I \in D_{0}}\left[\sum_{J \subset I} \psi(J)-\psi(I)\right]^{-}<\epsilon .
$$

In an analogous fashion we can define quasi overadditive scalar functions. A scalar function $\psi$ is said to be quasi normal if it is either quasi additive, or quasi subadditive, or quasi overadditive.

We shall denote by $S(\phi, D)$ the sum $\sum \phi(I)$, where $\sum$ ranges over all $I \in D$. If $\psi$ is quasi subadditive, then $-\psi$ is quasi overadditive. It may occur that $\phi(I), \psi(I)$ depend also on arbitrary parameters, and we may require that the numbers $\eta(\epsilon), \lambda\left(\epsilon, D_{0}\right)$ of definitions $(\phi),(\psi)$ can be determined independently of the values chosen for these parameters.

(2.i) A vector function $\phi(I)=\left(\phi_{1}, \cdots, \phi_{k}\right), I \in\{I\}$, is quasi additive (with respect to $\delta(D)$ and $\mathfrak{D})$ if and only if all components $\phi_{r}(I), r=1, \cdots, k$, are quasi additive (with respect to $\delta(D)$ and $\mathfrak{D})$. 
This statement is an immediate consequence of the definitions.

(2.ii) If $\phi^{\prime}, \phi^{\prime \prime}$ are quasi additive, then the sum $\alpha \phi^{\prime}+\beta \phi^{\prime \prime}, \alpha, \beta$ real, is quasi additive. If $\phi^{\prime}, \phi^{\prime \prime}$ are quasi subadditive, then the sum $\alpha \phi^{\prime}+\beta \phi^{\prime \prime}, \alpha, \beta \geqq 0$, is quasi subadditive.

This statement is an immediate consequence of the definitions and of the triangular relations: $\|u+v\| \leqq\|u\|+\|v\|$ for all vectors $u, v,[u+v]^{+} \leqq u^{+}$ $+v^{+},[u+v]^{-} \leqq u^{-}+v^{-}$for all real numbers $u, v$. As a consequence of the definitions we have also

(2.iii) If all $\left|\phi_{r}\right|, r=1, \cdots, k$, are quasi additive, then also $\|\phi\|$ is quasi additive.

(2.iv) Under the conditions of $(\phi)$ we have $\left\|S(\phi, D)-S\left(\phi, D_{0}\right)\right\|<2 \epsilon$. For a nonnegative $\psi$, under the conditions of $(\psi)$, we have $S(\psi, D)-S\left(\psi, D_{0}\right)>-\epsilon$.

Proof. We have

$$
S(\phi, D)-S\left(\phi, D_{0}\right)=\sum_{I \in D}\left[\sum_{J \subset I} \phi(J)-\phi(I)\right]+\sum_{J \notin I} \phi(J),
$$

and $\left(\phi_{1}\right),\left(\phi_{2}\right)$ imply the first part of (2.iv). Analogous relation holds for $\psi$, and, if $\psi \geqq 0$, then $\left(\psi_{1}\right)$ implies the second part of (2.iv). finite

(2.v) If $\phi(I)$ is any quasi additive vector function, then the limit exists and is

$$
\begin{aligned}
& \mathfrak{B}=\mathfrak{B}(\phi)=\lim _{\delta(D) \rightarrow 0} S(\phi, D)=\lim _{\delta(D) \rightarrow 0} \sum_{I \in D} \phi(I), \\
& \mathfrak{B}=\left(\mathfrak{B}_{1}, \cdots, \mathfrak{B}_{k}\right),-\infty<\mathfrak{B}_{r}<+\infty, \quad r=1, \cdots, k .
\end{aligned}
$$

If $\psi(I)$ is any nonnegative quasi subadditive scalar function, then the limit exists (finite, or $+\infty$ )

$$
V(\psi)=\lim _{\delta(D) \rightarrow 0} S(\psi, D)=\lim _{\delta(D) \rightarrow 0} \sum_{I \in D} \psi(I),
$$

$0 \leqq V \leqq+\infty$. In other words, $\mathfrak{B}(\phi), V(\psi)$ exist as Burkill-type integrals.

Proof. Given $\epsilon>0$, let $\eta, D_{0}, \lambda$ as in $(\phi)$ and suppose $D, D^{\prime} \in \mathfrak{D}, \delta(D)<\lambda$, $\delta\left(D^{\prime}\right)<\lambda$. Then, by (2.iv), we have $\left\|S(\phi, D)-S\left(\phi, D_{0}\right)\right\|<2 \epsilon,\left\|S\left(\phi, D^{\prime}\right)-S\left(\phi, D_{0}\right)\right\|$ $<2 \epsilon$, and finally $\left\|S(\phi, D)-S\left(\phi, D^{\prime}\right)\right\|<4 \epsilon$. This proves the first part of (2.v). Given $\epsilon>0$ let $\eta$ be as in $(\psi)$ and note that, by the definition of ${ }^{*} V={ }^{*} V(\psi)$, there is a $D_{0} \in \mathfrak{D}$ with $\delta\left(D_{0}\right)<\eta, S\left(\psi, D_{0}\right)>^{*} V-\epsilon$ if ${ }^{*} V<+\infty,>\epsilon^{-1}$ if $* V=+\infty$. Let $\lambda=\lambda\left(\epsilon, D_{0}\right)$ as in $(\psi)$ and note that for every $D \in \mathcal{D}$ with $\delta(D)<\lambda$ we have, by (2.iv), $S(\psi, D)-S\left(\psi, D_{0}\right)>-\epsilon$ and hence $S(\psi, D)>* V$ $-2 \epsilon$ if $* V=+\infty,>\epsilon^{-1}-\epsilon$ if $* V=+\infty$. Thus $* V \geqq * V-2 \epsilon$, or $\geqq \epsilon^{-1}-\epsilon$ respectively, and finally $* V \geqq * V$. Thus, $* V=* V=V, 0 \leqq V \leqq+\infty$. 
Remark. Note that $V(\psi)$ is the lim $\sup S(\psi, D)$ and not the sup $S(\psi, D)$. Examples show (\$4) that these numbers may be different. If axiom $(\psi)$ is replaced by $\left(\psi^{*}\right)$ Given $\epsilon>0$ and any finite system $D_{0}=[I] \in \mathfrak{D}$, there is a number $\lambda=\lambda\left(\epsilon, D_{0}\right)>0$ such that for every system $D=[J] \in D$ with $\delta(D)<\lambda$ we have $\left(\psi_{1}\right)$, then $V(\psi)=\sup S(\psi, D)$.

\section{First properties of quasi normal functions.}

(3.i) If $\psi$ is any nonnegative quasi subadditive scalar function and $V(\psi)$ $<+\infty$, then $\psi$ is quasi additive. In other words, if (d), $(\psi)$ hold, $\psi \geqq 0$, and the Burkill-type integral $V(\psi)$ is finite, then $(\phi)$ holds.

Proof. Let $\eta(\epsilon), \lambda\left(\epsilon, D_{0}\right)$ be as in $(\psi)$. Since $V(\psi)$ exists and is finite, given $\epsilon>0$ there is a number $\mu(\epsilon)>0$ such that for every $D_{0}=[I], D_{0} \in \mathcal{D}$, with $\delta\left(D_{0}\right)<\mu(\epsilon)$ we have

$$
\left|V(\psi)-\sum_{I} \psi(I)\right|<\epsilon / 3,
$$

where $\sum_{I}$ denotes any sum ranging over all $I \in D_{0}$. Let $D_{0}$ be chosen in such a way that $\delta\left(D_{0}\right)<\min [\mu(\epsilon), \eta(\epsilon / 6)]$. Take $\lambda^{\prime}=\lambda^{\prime}(\epsilon)=\min \left[\mu(\epsilon), \lambda\left(\epsilon / 6, D_{0}\right)\right]$ and consider any system $D=[J], D \in \mathfrak{D}$, with $\delta(D)<\lambda^{\prime}$. By $(\psi)$ we have

$$
\sum_{I}\left[\sum^{(I)} \psi(J)-\psi(I)\right]^{-}<\epsilon / 6,
$$

where $\sum^{(I)}$ denotes any sum ranging over all $J \in D, J \subset I$. On the other hand, we have

$$
\left|V(\psi)-\sum_{J} \psi(J)\right|<\epsilon / 3,
$$

where $\sum_{J}$ denotes any sum ranging over all $J \in D$. Note that for every real $m$ we have $|m|=m^{+}+m^{-}, m=m^{+}-m^{-}$and hence $|m|=m+2 m^{-}$. We shall denote by $\Sigma^{\prime}$ any sum ranging over all $J \in D$ with $J \subset I$ for any $I \in D_{0}$. We have

$$
\begin{aligned}
0 & \leqq \sum_{I}\left|\sum^{(I)} \psi(J)-\psi(I)\right|+\sum^{\prime} \psi(J) \\
& =\sum_{I}\left[\sum^{(I)} \psi(J)-\psi(I)\right]+2 \sum_{I}\left[\sum^{(I)} \psi(J)-\psi(I)\right]^{-}+\sum^{\prime} \psi(J) \\
& =\left[\sum_{J} \psi(J)-V(\psi)\right]-\left[\sum_{I} \psi(I)-V(\psi)\right]+2 \sum_{I}\left[\sum^{(I)} \psi(J)-\psi(I)\right]^{-},
\end{aligned}
$$

where in the second member both expressions are nonnegative. By (3.1), (3.2) and (3.3) we have

$$
0 \leqq \sum_{I}\left|\sum^{(I)} \psi(J)-\psi(I)\right|+\sum^{\prime} \psi(J) \leqq \epsilon / 3+\epsilon / 3+\epsilon / 3=\epsilon,
$$

and both requirements $\left(\phi_{1}\right),\left(\phi_{2}\right)$ are satisfied for the nonnegative scalar function $\psi$, i.e., $\psi$ is quasi additive.

(3.ii) If $\phi(I)=\left(\phi_{1}, \cdots, \phi_{n}\right)$ is quasi additive, then the functions $\phi_{r}(I)$, $r=1, \cdots, k$, are also quasi additive, and the functions $\|\phi\|,\left|\phi_{r}\right|, \phi_{r}^{+}, \phi_{r}^{-}$are quasi subadditive. 
Proof. We shall use below the notations $\sum_{I}, \sum^{(I)}, \sum^{\prime}$ introduced for the proof of (3.i). Given $\epsilon>0$, let $\eta(\epsilon), D_{0}=[I], \lambda\left(\epsilon, D_{0}\right), D=[J]$ as in $(\phi)$. For every $I \in D_{0}$ let $u(I)=\left(u_{1}, \cdots, u_{k}\right)$ be the vector

$$
u(I)=\sum^{(I)} \phi(J)-\phi(I) .
$$

Then, by Minkowski's inequality, we have

$$
\begin{aligned}
\|\phi(I)+u(I)\| & =\left\|\sum^{(I)} \phi(J)\right\|_{=}=\left[\sum_{r}\left(\sum^{(I)} \phi_{r}(J)\right)^{2}\right]^{1 / 2} \\
& \leqq \sum^{(I)}\left[\sum_{r} \phi_{r}^{2}(J)\right]^{1 / 2}=\sum^{(I)}\|\phi(J)\| .
\end{aligned}
$$

On the other hand, $\phi(I)=[\phi(I)+u(I)]+[-u(I)]$, and hence

$$
\begin{aligned}
\sum^{(I)}\|\phi(J)\|-\|\phi(I)\| & \geqq\|\phi(I)+u(I)\|-\|\phi(I)\| \geqq-\|u(I)\|, \\
{\left[\sum^{(I)}\|\phi(J)\|-\|\phi(I)\|\right]^{-} } & \leqq u(I) \|, \\
\sum_{I}\left[\sum^{(I)}\|\phi(J)\|-\|\phi(I)\|\right]^{-} & \leqq \sum_{I}\|u(I)\|<\epsilon,
\end{aligned}
$$

and this proves that $\|\phi\|$ is quasi subadditive. By (2.i) we know that the functions $\phi_{r}, r=1, \cdots, k$, are quasi additive, and by the result now proved we conclude that the functions $\left|\phi_{r}\right|$ are quasi subadditive.

Observe that for all real numbers $m, p$ with $p \geqq 0$ we have $|m-p|=|p-m|$ $\geqq m^{-}$. Indeed, if $m \geqq 0$, then $m^{-}=0$; if $m \leqq 0$, then $|m-p|=\left|-m^{-}-p\right|=m^{-}$ $+p \geqq m^{-}$. We have now

$$
\begin{aligned}
u_{r}(I) & =\sum^{(I)} \phi_{r}(J)-\phi_{r}(I) \\
& =\left[\sum^{(I)} \phi_{r}^{+}(J)-\phi_{r}^{+}(I)\right]-\left[\sum^{(I)} \phi_{r}^{-(J)}-\phi_{r}^{-}(I)\right] .
\end{aligned}
$$

If $\phi_{r}(I) \geqq 0$ then $\phi_{r}^{-}(I)=0$ and

$$
\left[\sum^{(I)} \phi_{r}^{+}(J)-\phi_{r}^{+}(I)\right]^{-} \leqq\left|u_{r}(I)\right|, \quad\left[\sum^{(I)} \phi_{r}^{-}(J)-0\right]^{-}=0 .
$$

If $\phi_{r}(I) \leqq 0$, then $\phi_{r}^{+}(I)=0$ and

$$
\left[\sum^{(I)} \phi_{r}^{+}(J)-0\right]^{-}=0, \quad\left[\sum^{(I)} \phi_{r}^{-}(J)-\phi_{r}^{-}(I)\right]^{-} \leqq\left|u_{r}(I)\right| .
$$

In both cases we have

$$
\left[\sum^{(I)} \phi_{r}^{+}(J)-\phi_{r}^{+}(I)\right]^{-} \leqq\left|u_{r}(I)\right|, \quad\left[\sum^{(I)} \phi_{r}^{-}(J)-\phi_{r}^{-}(I)\right]^{-} \leqq\left|u_{r}(I)\right|,
$$

and finally, since $\phi_{r}$ is quasi additive, also

$$
\sum_{I}\left[\sum^{(I)} \phi_{r}^{+}(J)-\phi_{r}^{+}(I)\right]^{-}, \quad \sum_{I}\left[\sum^{(I)} \phi_{r}^{-}(J)-\phi_{r}^{-}(I)\right]^{-} \leqq \sum_{I}\left|u_{r}(I)\right|<\epsilon .
$$

Thus $\phi_{r}^{+}, \phi_{r}^{-}$are quasi subadditive, and (3.ii) is proved.

(3.iii) If all components $\phi_{r}$ of $\phi=\left(\phi_{1}, \cdots, \phi_{k}\right)$ are nonnegative and quasi subadditive, then $\|\phi\|$ is quasi subadditive. If all functions $\phi_{r}^{+}, \phi_{r}^{-}, r=1, \cdots, k$, are quasi additive, then all functions $\phi_{r},\left|\phi_{r}\right|,\|\phi\|$ are quasi additive.

Proof. Given $\epsilon>0$, there is $\eta(\epsilon)>0$ such that for every $D_{0}=[I]$ with $\delta\left(D_{0}\right)$ $\left\langle\eta\right.$, there is a $\lambda=\lambda\left(\epsilon, D_{0}\right)>0$ such that for $D=[J]$ with $\delta(D)<\lambda$ we have 


$$
\sum_{r}\left[\sum^{(I)} \phi_{r}(J)-\phi_{r}(I)\right]^{-}<\epsilon,
$$$$
r=1, \cdots, k \text {. }
$$

If $u_{r}, u_{r}^{+}, u_{r}^{-}$have the usual meaning, we have $u_{r}=u_{r}^{+}-u_{r}^{-}, u_{r}^{+} \geqq 0, u_{r}^{-} \geqq 0$, and

$$
0 \leqq \sum_{r} u_{r}^{-}(I)<\epsilon
$$$$
r=1, \cdots, k \text {. }
$$

If $u^{+}(I)=\left(u_{1}^{+}, \cdots, u_{k}^{+}\right), u^{-}(I)=\left(u_{1}^{-}, \cdots, u_{k}^{-}\right)$, then, as above, we have

$$
\begin{aligned}
\|\phi(I)+u(I)\| & \leqq \sum^{(I)}\|\phi(J)\|, \\
\|\phi(I)\| & \leqq\left\|\phi(I)-u^{-}(I)\right\|+\left\|u^{-}(I)\right\| .
\end{aligned}
$$

If $w(I)=\left(w_{1}, \cdots, w_{k}\right)$ is the vector whose components are $w_{r}=0$ if $\phi_{r}-u_{r}^{-}$ $\geqq 0, w_{r}=\left|\phi_{r}-u_{r}\right|$ if $\phi_{r}-u_{r}<0$, then, since $\phi_{r} \geqq 0$, we have, in the latter case, $\left|w_{r}\right|=\left|\phi_{r}-u_{r}^{-}\right| \leqq\left|u_{r}^{-}\right|=u_{r}^{-}$, and in any case, $\left|w_{r}\right| \leqq u_{r}^{-}, r=1, \cdots, k$. Hence $\|w\| \leqq\left\|u^{-}\right\|$, and also

$$
\left\|\phi(I)-u^{-}(I)\right\| \leqq\left\|\phi(I)-u^{-}(I)+w(I)\right\|+\|w(I)\| .
$$

Finally, we observe that all components of the vectors $\phi-u^{-}+w$ and $\phi-u^{-}$ $+w+u^{+}$are not negative, and the components of the former are $\leqq$the corresponding components of the latter. Hence

$$
\left\|\phi-u^{-}+w\right\| \leqq\left\|\phi-u^{-}+w+u^{+}\right\|=\|\phi+u+w\| .
$$

By force of (3.4), (3.5), (3.6) we have successively,

$$
\begin{aligned}
\|\phi\| & \leqq\left\|\phi-u^{-}\right\|+\left\|u^{-}\right\| \leqq\left\|\phi-u^{-}+w\right\|+\|w\|+\left\|u^{-}\right\| \\
& \leqq\|\phi+u+w\|+\|w\|+\left\|u^{-}\right\| \leqq\|\phi+u\|+\|w\|+\|w\|+\left\|u^{-}\right\| \\
& \leqq\|\phi+u\|+3\left\|u^{-}\right\|, \\
\|\phi(I)\| & \leqq\|\phi(I)+u(I)\|+3\left[u_{1}^{-}(I)+\cdots+u_{\bar{k}}(I)\right] .
\end{aligned}
$$

Finally, we have

$$
\begin{aligned}
\|\phi(I)\| & \leqq \sum^{(I)}\left\|_{\phi(J)}\right\|+3 \sum_{r} u_{r}^{-}(I), \\
\sum_{I}\left[\sum^{(I)}\left\|_{\phi(J)}\right\|-\|\phi(I)\|\right]^{-} & \leqq 3 \sum_{r} \sum_{I} u_{r}^{-}(I) \leqq 3 k \epsilon^{2} .
\end{aligned}
$$

Thus, the first part of (3.iii) is proved. To prove the last part, observe that $\phi_{r}=\phi_{r}^{+}-\phi_{r}^{-},\left|\phi_{r}\right|=\phi_{r}^{+}+\phi_{r}^{-}$are quasi additive by force of (2.ii), and that $\phi,\|\phi\|$ are quasi additive by force of (2.i) and (2.iii).

(3.iv) If $\phi$ is any quasi additive vector function, then the limits exist

$$
\begin{aligned}
\mathfrak{B}=\mathfrak{B}(\phi) & =\lim _{\delta(D) \rightarrow 0} S(\phi, D), & \mathfrak{B}=\left(\mathfrak{B}_{1}, \cdots, \mathfrak{B}_{k}\right), \\
\mathfrak{B}_{r}=\mathfrak{B}\left(\phi_{r}\right) & =\lim _{\delta(D) \rightarrow 0} S\left(\phi_{r}, D\right), & -\infty<\mathfrak{B}_{r}<+\infty, r=1, \cdots, k, \\
V=V(\|\phi\|) & =\lim _{\delta(D) \rightarrow 0} S(\|\phi\|, D), & 0 \leqq V \leqq+\infty,
\end{aligned}
$$




$$
\begin{array}{cl}
V_{r}=V\left(\left|\phi_{r}\right|\right)=\lim _{\delta(D) \rightarrow 0} S\left(\left|\phi_{r}\right|, D\right), & 0 \leqq V_{r} \leqq+\infty, r=1, \cdots, k, \\
V_{r}^{+}=V\left(\phi_{r}^{+}\right)=\lim _{\delta(D) \rightarrow 0} S\left(\phi_{r}^{+}, D\right), & 0 \leqq V_{r}^{+} \leqq+\infty, r=1, \cdots, k, \\
V_{r}^{-}=V\left(\phi_{r}^{-}\right)=\lim _{\delta(D) \rightarrow 0} S\left(\phi_{r}^{-}, D\right), & 0 \leqq V_{r}^{-} \leqq+\infty, r=1, \cdots, k .
\end{array}
$$

This statement is a consequence of (3.ii), (2.v). Note that, if $V=V(\|\phi\|)$ $<+\infty$, then $V_{r}, V_{r}^{+}, V_{r}^{-}<+\infty$. have

(3.v) If $\phi$ is any quasi additive vector set function and $V(\|\phi\|)<+\infty$, we

$$
\begin{aligned}
& V_{r}^{+}-V_{r}^{-}=\mathfrak{B}_{r}, \quad V_{r}^{+}+V_{r}^{-}=V_{r}, \\
& \left|\mathfrak{B}_{r}\right| \leqq V_{r} \leqq V, \quad \quad r=1, \cdots, k \\
& \|\mathfrak{B}\|=\left(\sum_{r=1}^{k} \mathfrak{B}_{r}^{2}\right)^{1 / 2} \leqq\left(\sum_{r=1}^{k} V_{r}^{2}\right)^{1 / 2} \leqq\|V\| \leqq \sum_{r=1}^{k} V_{r} .
\end{aligned}
$$

These inequalities are an immediate consequence of the definitions. Note that the third inequality of the last line is a consequence of the triangular relation

$$
\left\{\sum_{r=1}^{k}\left[\sum_{I \in D}\left|\phi_{r}(I)\right|\right]^{2}\right\}^{1 / 2} \leqq \sum_{I \in D}\left[\sum_{r=1}^{k} \phi_{r}^{2}(I)\right]^{1 / 2} .
$$

(3.vi) If $\phi$ is any quasi additive set function and $V(\|\phi\|)<+\infty$, then all functions $\phi, \phi_{r},\|\phi\|,\left|\phi_{r}\right|, \phi_{r}^{+}, \phi_{r}^{-}$are quasi additive.

Indeed, all $\phi_{r}$ are quasi additive by (2.i) and $\|\phi\|,\left|\phi_{r}\right|, \phi_{r^{+}}, \phi_{r}^{-}$are quasi subadditive by (3.ii). By $V<+\infty$ we deduce $V_{r}, V_{r}^{+}, V_{r}^{-}<\infty$, and by (3.i) we conclude that $\|\phi\|,\left|\phi_{r}\right|, \phi_{r}^{+}, \phi_{r}^{-}$are quasi additive.

(3.vii) If $\phi$ is quasi additive and $V(\|\phi\|)<+\infty$, then given $\epsilon>0$ there is a number $\mu(\epsilon)>0$ such that, for every $D_{0}=[I], D_{0} \in \mathfrak{D}$, with $\delta\left(D_{0}\right)<\mu$, we have

$$
\left\|\mathfrak{B}(\phi)-\sum_{I \in D_{0}} \phi(I)\right\|<\epsilon, \quad \mid V(\|\phi\|)-\sum_{I \in D_{0}}\|\phi(I)\|<\epsilon,
$$

and analogous relations hold for $V_{r}, V_{r}^{+}, V_{r}^{-}, r=1, \cdots, k$. In addition, given $\epsilon>0$ and $D_{0} \in \mathfrak{D}$ with $\delta\left(D_{0}\right)<\mu(\epsilon)$, there is another number $\lambda=\lambda\left(\epsilon, D_{0}\right), 0<\lambda \leqq \mu$, such that for every other system $D=[J], D \in \mathfrak{D}$, with $\delta(D)<\lambda\left(\epsilon, D_{0}\right)$, we have

$$
\begin{aligned}
& \sum_{I \in D_{0}}\left\|\sum_{J \in I} \phi(J)-\phi(I)\right\|<\epsilon, \quad \sum_{I \in D_{0}}\left|\sum_{J \subset I}\|\phi(J)\|-\|\phi(I)\|\right|<\epsilon, \\
& \sum_{J \notin I \text { for any } I \in D_{0}}\|\phi(J)\|<\epsilon,
\end{aligned}
$$


and analogous relations hold for $\phi_{r}, \phi_{r}^{+}, \phi_{r}^{-}$. We may also suppose $\mu(\epsilon) \leqq \epsilon$.

This statement is a consequence of $\left(\phi_{1}\right),\left(\phi_{2}\right)$, (3.iv), and (3.vi).

4. Examples.

1. Suppose $A=[0,1]$ with the usual topology, $\{I\}$ the class of all intervals $I=[a, b] \subset A, a<b$, and define $|I|=b-a$. Suppose $\mathfrak{D}$ be the family of all finite systems $D=[I]$ of nonoverlapping $I \in\{I\}$. If we take $\delta(D)$ $=\left[1-\sum|I|\right]+\max |I|$, then $0<\delta(D)<2$, and the axioms (d) are trivial. Take $k=1$ and $\phi(I)=b-a>0$. Given $\epsilon>0$ arbitrary take $\eta=\eta(\epsilon)=\epsilon / 2$ and let $D_{0}=\left[I_{j}, j=1, \cdots, N\right]$ be any system $D_{0} \in \mathcal{D}$ with $\delta\left(D_{0}\right)<\eta$. Take $\lambda=\lambda\left(\epsilon, D_{0}\right)=\epsilon / 4 N$. If $D=[J]$ is any system with $\delta(D)<\lambda$, and we denote by $\sum^{\prime}$ any sum ranging over all $J \in D$ with $J \nsubseteq I$ for any $I \in D_{0}$, we have $\sum^{\prime} \phi(J)<\left[1-\sum\left|I_{j}\right|\right]+2 N(\epsilon / 4 N)<\epsilon / 2+\epsilon / 2=\epsilon$, and $\left(\phi_{2}\right)$ holds. Also, if $\sum{ }^{(j)}$ denotes a sum ranging over all $J \in D$ with $J \subset I_{j}$, we have $\phi\left(I_{j}\right)$ $-\sum{ }^{(j)} \phi(J)>0$, and $\sum_{j}\left[\phi\left(I_{j}\right)-\sum{ }^{(j)} \phi(J)\right]<\left[1-\sum|J|\right]+2 N(\epsilon / 4 N)<\epsilon / 2$ $+\epsilon / 2=\epsilon$, and $\left(\phi_{1}\right)$ holds. Thus, $\phi$ is quasi additive with $\mathfrak{B}=1$.

2. Suppose $A,\{I\}$, and $\mathfrak{D}$ as above. Take $\phi(I)=|I|=b-a$ if $I=[a, b]$, $0<a<b \leqq 1, \phi(I)=|I|+1$ if $0=a<b \leqq 1$. Take $\delta(D)$ as above if for no $I \in D$ we have $a=0$, take $\delta(D)=\left[1-\sum|I|\right]+\max |I|+1$ if for one $I \in D$ we have $I=[0, b]$. If $\eta<1$ and $\delta(D)<1, D=[I]$, then there is in $D$ no interval $I=[0, b]$. Axioms (d) are trivial. By the same reasoning above with $\eta=\min [\epsilon / 2,1]$ we prove that $\phi$ is quasi additive with $V=1$.

3. The same as 2 with the two definitions of $\delta(D)$ exchanged. Then if $\eta<1$ and $\delta(D)<1, D=[I]$, there is in $D$ one interval $I=[0, b]$. By the same reasoning we prove that $\phi$ is quasi additive with $V=2$.

4. Suppose $A,\{I\}$, and $\mathfrak{D}$ as above. For every real $m, 0 \leqq m \leqq 1$, take $\sigma(m)=0$ if $m$ is irrational, $\sigma(m)=1 / p$ if $m=q / p, p, q$ integers, $p \geqq 1$ minimum, $0 \leqq q \leqq p$. Take $k=1$ and $\phi(I)=|I|=b-a$ for $I=[a, b] \subset A$. For $D=\left[I_{j}, j=1, \cdots, N\right], I_{j}=\left[a_{j}, b_{j}\right]$ take $\delta(D)=\left[1-\sum\left|I_{j}\right|\right]+\max \left|I_{j}\right|$ $+\sum \sigma\left(a_{j}\right)+\sum \sigma\left(b_{j}\right)$. For $D=\left[I_{1}\right], I_{1}=[0,1]$, we have $\delta(D)=0+1+1+1=3$; for $D=\left[I_{1}, I_{2}\right], I_{1}=[0,1 / 2], I_{2}=[1 / 2,1]$, we have $\delta(D)=1 / 2+(1+1 / 2)$ $+(1 / 2+1)=3+1 / 2$. Thus $\delta(D)$ does not necessarily decrease by refinement. Axiom $\left(\mathrm{d}_{1}\right)$ is trivial. Given $\epsilon>0$ let $D=\left[I_{j}\right]$ with $I_{j}=\left[a_{j}, b_{j}\right]$, all $a_{j}, b_{j}$ irrational with $1-\sum\left(b_{j}-a_{j}\right)<\epsilon / 2, b_{j}-a_{j}<\epsilon / 2$. Then $\delta(D)<\epsilon$. We may also choose an integer $M \geqq 3, M>4 / \epsilon, M$ prime, and suppose $a_{j}, b_{j}$ all of the form $q / M^{2}, q=1,2, \cdots, M^{2}-1$, and $N=M$. Namely, we may take $a_{1}=1 / M^{2}$, $a_{2}=b_{1}=1 / M+1 / M^{2}, a_{3}=b_{2}=2 / M+1 / M^{2}, \cdots, a_{N}=b_{N-1}=(M-1) / M$ $+1 / M^{2}, b_{N}=1-1 / M^{2}$. Then $\delta(D)=\left(2 / M^{2}\right)+(1 / M)+M\left(2 / M^{2}\right)<4 / M<\epsilon$. This remark shows that Axiom $\left(\mathrm{d}_{2}\right)$ also holds. The proof that the Axioms $\left(\phi_{1}\right),\left(\phi_{2}\right)$ hold is the same as for Example 1. Thus $\phi$ is quasi additive and $\mathfrak{B}=1$.

5. Suppose $A,\{I\}$, and $\mathfrak{D}$ as above. Suppose $k=1$ and $\phi(I)=|I|=b-a$ if both $a, b$ are irrational, $\phi(I)=a-b$ otherwise. For $D=[I] \in \mathfrak{D}, I=[a, b]$, 
take $\delta(D)=\left(1-\sum|I|\right)+\max |I|$ if all $a, b$ are irrational, take $\delta(D)=1$ otherwise. Obviously Axioms (d) are satisfied. If $D=[I], \delta(D)<1$, then all $a$ and $b$ are irrational. Obviously Axioms $(\phi)$ are satisfied, $\phi(I)$ is quasi additive, and $\mathfrak{B}=1$.

6. The same as in 5 , with $\delta(D)=\left(1-\sum|I|\right)+\max |I|$ if all $a, b$ are rational, $\delta(D)=1$ otherwise. All Axioms (d) and $(\phi)$ are satisfied, $\phi$ is quasi additive, and $\mathfrak{B}=-1$.

7. Jordan length for continuous curves. Let $C: x=x(t), 0 \leqq t \leqq 1$, $x=\left(x_{1}, \cdots, x_{k}\right)$, be any real continuous vector function (a continuous parametric curve). Take $A=[0,1],\{I\}$ the class of all intervals $I=[a, b] \subset A$, $\mathfrak{D}$ the class of all finite subdivisions $D=\left[I_{j}, j=1, \cdots, N\right], I_{j}=\left[a_{j-1}, a_{j}\right]$, $0=a_{0}<a_{1}<\cdots<a_{N}=1$, and suppose $\delta(D)=\max \left|I_{j}\right|=\max \left(a_{j}-a_{j-1}\right)$. Then $0<\delta(D) \leqq 1$ and Axioms (d) are trivial. Suppose $\phi(I)=\left(\phi_{1}, \cdots, \phi_{k}\right)$, $\phi_{r}(I)=x_{r}(b)-x_{r}(a), r=1, \cdots, k$, for every $I=[a, b] \subset A$. Denote by $M_{r}=\max \left|x_{r}(t)\right|$ for $0 \leqq t \leqq 1$, and $M=\max M_{r}, r=1, \cdots, k$. Given $\epsilon>0$ take $\eta=\eta(\epsilon)=2$, let $D_{0}=\left[I_{i}, i=1, \cdots, N\right]$ be any system $D_{0} \in \mathfrak{D}$ (with $\left.\delta\left(D_{0}\right)<2\right)$. Because of the continuity of $x(t)$ there is a $\lambda=\lambda\left(\epsilon, D_{0}\right)>0$ such that $\|\phi(I)\|<\epsilon / 2 N$ for all $I=[a, b]$ with $b-a<\lambda$. Now suppose $D=[J]$ be any system $D \in D$ with $\delta(D)<\lambda$. Then the sum $\sum^{\prime}\|\phi(J)\|$ contains at most $2(N-1)$ terms and $\sum^{\prime}\|\phi(J)\|<2(N-1)(\epsilon / 2 N)<\epsilon$. For each $I_{i} \in D_{0}$ the intervals $J \in D, J \subset I_{i}$, leave uncovered in $I$ at most two terminal intervals, say $H^{\prime}, H^{\prime \prime}$ (if any). Then we have

$$
\begin{aligned}
\phi\left(I_{i}\right) & =\sum^{(i)} \phi(J)+\phi\left(B^{\prime}\right)+\phi\left(B^{\prime \prime}\right), \\
\left\|\phi\left(I_{i}\right)-\sum^{(i)} \phi(J)\right\| & \leqq\left\|\phi\left(B^{\prime}\right)\right\|+\left\|\phi\left(B^{\prime \prime}\right)\right\|<\epsilon / N,
\end{aligned}
$$

and

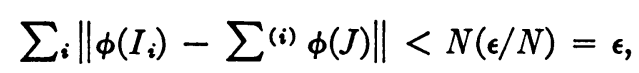

where $\sum_{i}$ ranges over all $i=1, \cdots, N$, and $\sum^{(i)}$ over all $J \in D, J \subset I_{i}$. Thus $\phi$ is quasi additive, and by (2.i) all $\phi_{r}$ are quasi additive. By (3.ii) all $\|\phi\|$, $\left|\phi_{r}\right|, \phi_{r}^{+}, \phi_{r}^{-}$are quasi subadditive. Obviously $V=V(\|\phi\|)$ is the Jordan length of the curve $C$, and $V_{r}=V\left(\left|\phi_{r}\right|\right), V_{r}^{+}=V\left(\phi_{r}^{+}\right), V_{r}^{-}=V\left(\phi_{r}^{-}\right)$are the total variation, the positive and negative variations of $x_{r}(t), 0 \leqq t \leqq 1$. If $V<+\infty$, then $C$ is rectifiable, $V_{r}, V_{r}^{+}, V_{r}^{-}<+\infty$, and the functions $\phi, \phi_{r}$, $\|\phi\|,\left|\phi_{r}\right|, \phi_{r}^{+}, \phi_{r}^{-}$are all quasi additive.

8. Jordan length for discontinuous curves. Let $C: x=x(t), 0 \leqq t \leqq 1$, $x=\left(x_{1}, \cdots, x_{k}\right)$, be any real vector function (a parametric curve not necessarily continuous). Take $A,\{I\}, \mathfrak{D}$ as in Example 7. For every $t_{0}, 0 \leqq t_{0}<1$, let $s^{+}\left(t_{0}\right)=\lim \sup \left\|x(t)-x\left(t_{0}\right)\right\|$ as $t \rightarrow t_{0}+$, and let $s^{+}(1)=0$. Analogously, for every $t_{0}, 0<t_{0} \leqq 1$, let $s^{-}\left(t_{0}\right)=\lim \sup \left\|x(t)-x\left(t_{0}\right)\right\|$ as $t \rightarrow t_{0}-$, and let $s^{-}(0)=0$. Finally, let $s\left(t_{0}\right)=s^{+}\left(t_{0}\right)+s^{-}\left(t_{0}\right)$ for every $0 \leqq t_{0} \leqq 1$. As in Example 7, we suppose $\phi(I)=\left(\phi_{1}, \cdots, \phi_{k}\right), \phi_{r}(I)=x_{r}(b)-x_{r}(a), r=1, \cdots, k$, for every 
$I=[a, b] \subset A$. Then $V=\sup \sum\|\phi(I)\|$ is the Jordan length of $C$, and $V<+\infty$ if and only if $x_{r}(t), r=1, \cdots, k$, are $B V$ in $A$. If $V<+\infty$, then $s(t), 0 \leqq t \leqq 1$, is zero everywhere but for countably many $t$, and the sum (or the sum of the series) $\sigma=\sum s(t) \leqq V$ is finite, where $\sum$ ranges over all $t$. If we take $\delta(D)$ $=\max \left|I_{i}\right|+\sigma-\sum s\left(t_{i}\right)$, where $D=\left[I_{1}, \cdots, I_{N}\right], \quad I_{i}=\left[a_{i-1}, a_{i}\right], \quad 0=a_{0}$ $<a_{1}<\cdots<a_{N}=1$, where $\max$ is taken for all $i=1, \cdots, N$, and $\sum$ ranges over all $i=0,1, \cdots, N$, then $\delta(D)$ is a mesh function and $\phi(I), I \in\{I\}$ is quasi additive with respect to $\delta(D)$ and $\mathfrak{D}$. We leave the proof to the reader. Then it is easy to prove also that $V=V(\|\phi\|)=\lim \sum\|\phi(I)\|$ as $\delta(D) \rightarrow 0$ where $\sum$ ranges over all $I \in D, D \in \mathfrak{D}$.

9. Cauchy integral in an interval in $E_{m}$. We may suppose $A$ is the unit interval $A=\left[0 \leqq x_{r} \leqq 1, r=1, \cdots, m\right]$, and $\mathfrak{D}$ is the collection of all finite subdivisions $D=I$ of $A$ into intervals $I=\left[a_{r} \leqq x_{r} \leqq b_{r}, r=1, \cdots, m\right]$. Let $f(x), x \in A, x=\left(x_{1}, \cdots, x_{m}\right)$, be any bounded real function $|f(x)| \leqq M$, and, for every $I$, let $l=l(I), L=L(I)$ denote the infimum and supremum of $f(x)$ in $I, \Delta(I)=L-l, F=F(I)$ any number $l \leqq F \leqq L$, and $\phi(I)=F(I)|I|,|I|$ $=\left(b_{1}-a_{1}\right) \cdots\left(b_{m}-a_{m}\right)$. Let $\delta(D)=\max \operatorname{diam} I$ for all $I \in D$, where $D \in \mathfrak{D}$. Then $\delta(D)$ is a mesh. The Riemann integrability condition reads: (R) Given $\epsilon>0$ there is a $\sigma=\sigma(\epsilon)>0$ such that $\sum \Delta(I)|I|<\epsilon$ for all $D \in \mathfrak{D}$ with $\delta(D)<\sigma$. It is easy to verify that (R) implies the quasi additivity of $\phi(I)$ (with respect to $\mathfrak{D}$ and $\delta(D)$ ) and vice-versa. The limit $\mathfrak{B}=\lim \sum \phi(I)$ is the Cauchy integral of $f(x)$ in $A$.

10. Lebesgue-Stieltjes integral. Let $\mu$ be a measure function in a $\sigma$-ring $\mathfrak{B}$ of subsets of a space $A$, i.e., $(A, \mathfrak{B}, \mu)$ is a measure space, and let $f(x), x \in A$, be a $\mu$-measurable and $\mu$-integrable function. It is not restrictive to suppose $f(x) \geqq 0$. For every $0<p<+\infty$ and $0 \leqq p<q \leqq+\infty$, denote by $B(p), I(p, q)$ respectively the sets $B(p)=[x \in A, f(x)=p], I(p, q)=[x \in A, p<f(x) \leqq q]$. These sets are all $\mu$-measurable and $0 \leqq p \mu[B(p)]<+\infty, \mu[B(+\infty)]=0$, (and we define $p \mu[B(p)]=0$ for $p=+\infty), p \mu[I(p, q)]<+\infty$, and $\sum p \mu[B(p)]$ $\leqq M<+\infty$ for some $M$ (say $M \geqq 1$ ), where $\sum$ ranges over all $p>0$. Thus the set $P$ of all $p>0$ with $\mu[B(p)]>0$ is countable and the corresponding series $\sum p \mu[B(p)]$ is convergent. Also $I(p-\tau, p+\tau) \rightarrow B(p), \mu[I(p-\tau, p+\tau)]$ $\rightarrow \mu[B(p)]$ as $\tau \rightarrow 0+$ for every $p>0$. Let $\{I\}$ be the collection of all sets $I(p, q), 0 \leqq p<q \leqq+\infty$, and let $\psi(I)=p \mu[I(p, q)], I \in\{I\}$. Let $\mathcal{D}$ be the family of all finite decompositions $D=\left[I_{i}, i=1, \cdots, n\right]$ of $A$ into sets $I_{i}=\left[x \in A, p_{i-1}<f(x) \leqq p_{i}\right] \in\{I\}$ with $0=p_{0}<p_{1}<\cdots<p_{n-1}<p_{n}=+\infty$. Finally, let $\delta(D)=1$ if $n=1$, and, if $n>1$,

$$
\delta(D)=\max _{i=1, \ldots, n-1}\left[p_{i}-p_{i-1}\right]+\frac{1}{p_{n-1}}+\sum_{i=1}^{n-1} p_{i} \mu\left[B\left(p_{i}\right)\right] .
$$

Then $\delta(D)$ is a mesh function $(\S 1)$ and $\psi(I)$ is quasi additive (with respect to $\mathfrak{D}$ and $\delta(D))$. To prove the last statement, given $\epsilon>0$, take $\eta=\eta(\epsilon)$ 
$=\min [\epsilon / 4 M, 1]$ and let $D_{0}=\left[I_{i}=I\left(p_{i-1}, p_{i}\right), i=1, \cdots, N\right], 0=p_{0}<p_{1}$ $<\cdots<p_{N-1}<p_{N}=\infty$, be any $D_{0} \in \mathcal{D}$ with $\delta\left(D_{0}\right)<\eta$. Then there are numbers $\tau_{i}>0$ such that $\mu\left[I\left(p_{i}-\tau_{i}, p_{i}+\tau_{i}\right]<\mu\left[B\left(p_{i}\right)\right]+\epsilon / 4 N p_{i}, i=1, \cdots, N-1\right.$. Take $\tau=\min \tau_{i}$, and $\lambda=\lambda\left(\epsilon, D_{0}\right)=\min \left[p_{i}-p_{i-1}, i=1, \cdots, N-1 ; 1 / p_{N-1}\right.$; $\tau ; \eta]$. If $D=\left[J_{j}=\left(q_{j-1}, q_{j}\right), j=1, \cdots, n\right], 0=q_{0}<q_{1}<\cdots<q_{n-1}<q_{n}=+\infty$, is any $D \in \mathfrak{D}$ with $\delta(D)<\lambda$, we have $J_{1} \subset I_{1}, J_{n} \subset I_{N}$, and

$$
\begin{aligned}
\Delta & =\sum_{i=1}^{N}\left[\sum^{(i)} \psi\left(J_{j}\right)-\psi\left(I_{i}\right)\right]^{-} \\
& =\sum_{i=1}^{N}\left[\sum^{(i)} q_{j-1} \mu\left[J\left(q_{j-1}, q_{j}\right)\right]-p_{i-1} \mu\left[I\left(p_{i-1}, p_{i}\right)\right]\right]^{-}
\end{aligned}
$$

where all $q_{j-1}, q_{j}$ relative to $\sum^{(i)}$ are between $p_{i-1}$ and $p_{i}$, and $q_{0}=p_{0}=0$. We have

$$
\begin{aligned}
\Delta \leqq & \sum_{i=2}^{N} p_{i-1}\left[\sum^{(i)} \mu\left[J\left(q_{j-1}, q_{j}\right)\right]-\mu\left[I\left(p_{i-1}, p_{i}\right)\right]\right]^{-} \\
\leqq & \sum_{i=2}^{N-1} p_{i-1}\left[\mu\left[B\left(p_{i-1}\right)\right]+\epsilon / 4 N p_{i-1}+\mu\left[B\left(p_{i}\right)\right]+\epsilon / 4 N p_{i}\right] \\
& +p_{N-1}\left[\mu\left[B\left(p_{N-1}\right)\right]+\epsilon / 4 N p_{N-1}\right] \\
& <2 \delta\left(D_{0}\right)+\epsilon / 4+\epsilon / 4<\epsilon / 2+\epsilon / 4+\epsilon / 4=\epsilon .
\end{aligned}
$$

Thus $\psi(I), I \in\{I\}$, is quasi subadditive (with respect to $D$ and $\delta(D)$ ), and hence $V=V(\psi)=\lim \sum \psi(I)$ exists as $\delta(D) \rightarrow 0$, where $\sum$ ranges over all $I \in D$. Since $f(x)$ is $\mu$-integrable, we have $V=V(\psi)<+\infty$, and by (3.i), $\psi$ is quasi additive. Also it is easy to prove that $V(\psi)=(A) \int f(x) d \mu$ is the LebesgueStieltjes integral of $f(x)$ in $A$ (see, e.g., [5; 8]). (It is also known that the limit above exists even if taken as $\Delta \rightarrow 0$ with $\Delta=\max \left[p_{i}-p_{i-1}\right]+1 / p_{n-1}$.)

11. Weierstrass integral over a rectifiable continuous curve $C$. Let $C: x=x(t)$, $0 \leqq t \leqq 1, x=\left(x_{1}, \cdots, x_{k}\right)$, be any real continuous vector function with finite jordan length $L$. As in Example 7, let $A=[0,1], I$ the class of all $I=[a, b]$ $\subset A, D$ the class of all finite subdivisions $D=\left[I_{i}, i=1, \cdots, N\right], I_{i}=\left[a_{i-1}, a_{i}\right]$, $0=a_{0}<a_{1}<\cdots<a_{N}=1$, and $\delta=\delta(D)=\max \left(a_{i}-a_{i-1}\right)$, and $\phi(I)$ $=\left(\phi_{1}, \cdots, \phi_{k}\right), \phi_{r}(I)=x_{r}(b)-x_{r}(a)$. Then $L=V(\|\phi\|)$ (Example 7$)$ and all functions $\phi, \phi_{r},\|\phi\|,\left|\phi_{r}\right|, \phi_{r}^{+}, \phi_{r}^{-}$are quasi additive with respect to $D$ and $\delta(D)$. Let $K$ be any compact set containing the graph $[C]$ of $C$, i.e., $[C]$ $\subset K \subset E_{k}$, and let $f(p, q), p \in K, q \in E_{k}$, be any function continuous on $K \times E_{k}$ such that $f(p, t q)=t f(p, q)$ for all $t \geqq 0, p \in K, q \in E_{k}$. For every $I \in\{I\}$, let $\Phi(I)=f[x(\tau), \phi(I)]$, where $\tau$ denotes any arbitrary point $\tau \in I$. Then $\Phi(I)$, $I \in\{I\}$, is quasi additive with respect to $D$ and $\delta(D)$ by force of Theorem (6.i), and the numbers $\eta(\epsilon), \lambda\left(\epsilon, D_{0}\right)$ of $\S 2$, can be taken independently of the 
choice of the $\tau$ 's in the intervals $I$. Thus, the following limit exists and is finite:

$$
\begin{aligned}
\Im & =\int_{C} f(p, q)=\lim _{\delta(D) \rightarrow 0} \sum_{I \in D} \Phi(I) \\
& =\lim _{\delta(D) \rightarrow 0} \sum_{i=1}^{n} f\left[x\left(\tau_{i}\right), x\left(a_{i}\right)-x\left(a_{i-1}\right)\right],
\end{aligned}
$$

independently of the choices of the points $\tau_{i} \in\left[a_{i-1}, a_{i}\right]$. This limit is known as the Weierstrass integral of $f$ on the rectifiable curve $C$ and was studied by L. Tonelli, G. Bouligand, N. Aronszajn, K. Menger, C. Y. Pauc. See [1] for references. It is known that $\Im$ is invariant with respect to Fréchet equivalence, i.e., it is independent of the representation $C: x=x(t)$ of $C$. In particular, if $s=s(t)$ denotes the Jordan length of the curve $C_{t}$ defined by $x(t)$ on $[0, t]$, $0 \leqq s \leqq L$, and $X(s)=x[s(t)]$, then $C: x=x(s), 0 \leqq s \leqq L$, is a representation of $C, X(s)$ is Lipschitzian of constant 1 , and $\Im$ is given by the Lebesgue integral

$$
\Im=\int_{C} f(p, q)=\int_{0}^{L} f\left[X(s), X^{\prime}(s)\right] d s .
$$

12. Integral on a continuous surface of finite area. Let $A \subset E_{2}$ be any "admissible" set of the $w$-plane $E_{2}, w=(u, v)[1$, p. 27], thus $A$ may be, e.g., any open set, or any finitely connected closed Jordan region. Let $T: x=x(w)$, $w \in A, x=\left(x_{1}, x_{2}, x_{3}\right)$, be any continuous mapping (surface) from $A$ into any set $K$, thus, if $[T]$ is the graph of $T$, we have $[T] \subset K \subset E_{3}$. Note that $T$ is supposed to be single-valued but not necessarily one-one. Also, we suppose that the Lebesgue area $L=L(T)$ of $T$ is finite and positive. Let $\{\pi\}$ be the class of all closed simple polygonal regions $\pi \subset A$, and $\mathfrak{D}$ the class of all finite systems $D=[\pi]=\left[\pi_{1}, \cdots, \pi_{n}\right]$ of nonoverlapping regions $\pi \in\{\pi\}$. If $E_{2 r}$, $r=1,2,3$, denote the oriented coordinate planes of $E_{3}$, and $\tau_{r}$ the projection of $E_{3}$ onto $E_{2 r}$, then $T_{r}=\tau_{r} T$ is a plane continuous mapping from $A$ into $E_{2 r}$, whose Lebesgue area will be denoted by $L_{r}=L\left(T_{r}\right)$. Note that $T, T_{r}$ map the oriented boundary curve $\pi^{*}$ of $\pi$ into continuous closed curves $C, C_{r}$, and $C_{r}$ is the projection of $C$ on $E_{2 r}$. If $O\left(p, C_{r}\right), p \in E_{2 r}$, denotes the topological index of any point $p \in E_{2 r}$ with respect to $C_{r},\left(O=0\right.$ on $\left[C_{r}\right], O \geqslant 0$ or $=0$ on $\left.E_{2 r}-\left[C_{r}\right]\right)$, then the function $O\left(p, C_{r}\right), p \in E_{2 r}$, is $L$-integrable, and we put $u(\pi)=\left(u_{1}, u_{2}, u_{3}\right)$, $\pi \in\{\pi\}$, where $u_{r}(\pi)=\left(E_{2 r}\right) \int O\left(p, C_{r}\right), r=1,2,3$. In $[1$, p. 358] the following indices, or indices of fineness, have been defined

$$
\begin{array}{cr}
d(D)=\max \operatorname{osc}(T, \pi), & \pi \in D, \\
m(D)=\max m_{r}, & r=1,2,3, \\
\mu(D)=\max \left[L-\sum\|u(\pi)\|, L_{r}-\sum\left|u_{r}(\pi)\right|, r=1,2,3\right], &
\end{array}
$$


where $\sum$ ranges over all $\pi \in D$, and where $m_{r}=\left|B_{r}\right|$ is the two-dimensional Lebesgue measure of the compact set $B_{r}$,

$$
B_{r}=T_{r}\left(\bigcup_{r \in D} \pi^{*}\right)=\bigcup_{\pi \in D}\left[C_{r}\right], \quad r=1,2,3 .
$$

It is proved in $[1$, p. 358] that, given $\epsilon>0$, there are systems $D=[\pi]$ with $d, m, \mu<\epsilon$. Thus

$$
\delta(D)=d(D)+m(D)+\mu(D), \quad D \in \mathfrak{D},
$$

is a mesh function ( $\$ 1)$. The following statements have been proved:

(a) $[1, \mathrm{pp} .186,296]$. If $N\left(p, T_{r}\right)=\operatorname{Sup} \sum\left|O\left(p, C_{r}\right)\right|$, where Sup is taken for all $D \in \mathfrak{D}$, then $N\left(p, T_{r}\right), p \in E_{2 r}$, is $L$-integrable, and $L_{r}=\left(E_{2 r}\right) \int N\left(p, T_{r}\right)$, $r=1,2,3$.

(b) $\left[1\right.$, p. 358]. There are measurable sets $H_{r} \subset E_{2 r}$ such that $\sum\left|O\left(p, C_{r}\right)\right|$ $=N\left(p, T_{r}\right)$ for all $p \in E_{2 r}-H_{r}\left(\sum\right.$ ranges over all $\left.\pi \in D\right)$ and $\left|H_{r}\right| \leqq \mu$, $r=1,2,3$.

(c) Since $B_{r}$ is compact, if $B_{r \rho}, \rho>0$, denotes the $\rho$-neighborhood of $B_{r}$ in $E_{2 r}$, we have $B_{r \rho} \rightarrow B_{r},\left|B_{r \rho}\right| \rightarrow\left|B_{r}\right|$ as $\rho \rightarrow 0+$. Hence, there are numbers $\rho_{r}>0$ such that $\left|B_{r \rho}\right|<\left|B_{r}\right|+\sigma_{r}$ for $\rho \leqq \rho_{r}$, where $\sigma_{r}$ is any positive number, $r=1,2,3$.

(d) $[1$, p. 360]. If $D=[\pi]$ is any system as above, and $\delta=d+m+\mu$ is its mesh, if $D^{\prime}=\left[\pi^{\prime}\right]$ is any other system and $\delta^{\prime}=d^{\prime}+m^{\prime}+\mu^{\prime}$ is its mesh, if $d^{\prime}<\rho$, if $B_{r}, H_{r}, B_{r}^{\prime}, H_{r}^{\prime}$ are the sets defined above for $D$ and $D^{\prime}$ respectively, let $J_{r}$ be the set $J_{r}=B_{r p} \cup B_{r p}^{\prime} \cup H_{r} \cup H_{r}^{\prime}$. Then

$$
\begin{aligned}
\sum\left|\sum(\pi) u_{r}\left(\pi^{\prime}\right)-u_{r}(\pi)\right| & \leqq 2\left(J_{r}\right) \int N\left(p, T_{r}\right), & \\
\sum^{\prime}\left|u_{r}\left(\pi^{\prime}\right)\right| & <\left(J_{r}\right) \int N\left(p, T_{r}\right), & r=1,2,3,
\end{aligned}
$$

where $\sum$ ranges over all $\pi \in D, \sum(\pi)$ over all $\pi^{\prime} \in D^{\prime}$ with $\pi^{\prime} \subset \pi$, and $\sum^{\prime}$ over all $\pi^{\prime} \in D^{\prime}$ with $\pi^{\prime} \nsubseteq \pi$ for any $\pi \in D$.

From (d) it follows immediately that

$$
\begin{aligned}
\sum\left\|\sum^{(\pi)} u\left(\pi^{\prime}\right)-u(\pi)\right\| & <2 \sum_{r}\left(J_{r}\right) \int N\left(p, T_{r}\right), \\
\sum^{\prime}\left\|u\left(\pi^{\prime}\right)\right\| & <\sum_{r}\left(J_{r}\right) \int N\left(p, T_{r}\right),
\end{aligned}
$$

where $\sum_{r}$ ranges over $r=1,2,3$. We can now prove that $u(\pi)=\left(u_{1}, u_{2}, u_{8}\right)$, $\pi \in\{\pi\}$, is quasi additive with respect to $\mathfrak{D}$ and $\delta(D)$.

Indeed, given $\epsilon>0$, there are numbers $\sigma_{r}>0$ such that $(M) \int N\left(p, T_{r}\right)$ 
$<\epsilon / 30$ for any measurable set $M \subset E_{2 r}$ with $|M|<\sigma_{r}$. Take $\eta=\eta(\epsilon)=\sigma$ $=\min \left[\sigma_{1}, \sigma_{2}, \sigma_{3}\right]$, and suppose $D_{0}=[\pi] \in \mathfrak{D}$ with $\delta=\delta\left(D_{0}\right)=d+m+\mu<\eta$. Hence $\left|B_{r}\right|=m_{r} \leqq m \leqq \eta \leqq \sigma_{r}$, and, by (c), there are numbers $\rho_{r}>0$ such that $\left|B_{r \rho}\right|<\left|B_{r}\right|+\sigma_{r}$ for $\rho<\rho_{r}$, and finally $\left|B_{r \rho}\right| \leqq \sigma_{r}+\sigma_{r}=2 \sigma_{r}$. Also $\left|H_{r}\right| \leqq \mu \leqq \eta$ $\leqq \sigma_{r}$. Let $\rho_{0}=\min \left[\rho_{1}, \rho_{2}, \rho_{3}\right]$ and $\lambda=\lambda\left(\epsilon, D_{0}\right)=\min \left[\rho_{0}, \eta\right]$. If $D=\left[\pi^{\prime}\right] \in \mathfrak{D}$ with $\delta^{\prime}=\delta(D)=d^{\prime}+m^{\prime}+\mu^{\prime}<\lambda$, and $B_{r}^{\prime}, H_{r}^{\prime}$ are the relative sets, we have $\left|B_{r}^{\prime}\right|$ $\leqq m_{r}^{\prime} \leqq m^{\prime}<\lambda \leqq \eta \leqq \sigma_{r}$ and $\left|H_{r}^{\prime}\right| \leqq \mu^{\prime} \leqq \lambda \leqq \sigma_{r}$. Thus, if $I_{r}=B_{r p} \cup B_{r}^{\prime} \cup H_{r} \cup H_{r}^{\prime}$, we have $\left|J_{r}\right| \leqq 2 \sigma_{r}+\sigma_{r}+\sigma_{r}+\sigma_{r}=5 \sigma_{r}$, and $\left(J_{r}\right) \int N\left(p, T_{r}\right)<5(\epsilon / 30)=\epsilon / 6$, $r=1,2,3$. Thus

$$
\begin{gathered}
\sum\left\|\sum^{(\pi)} u\left(\pi^{\prime}\right)-u(\pi)\right\|<2 \cdot 3(\epsilon / 6)=\epsilon, \\
\sum^{\prime}\left\|u\left(\pi^{\prime}\right)\right\|<3(\epsilon / 6)<\epsilon .
\end{gathered}
$$

In other words, $u(\pi), \pi \in\{\pi\}$, is a quasi additive vector function (with respect to $\mathfrak{D}$ and $\delta(D))$, and

$$
V(\|\phi\|)=\lim _{\delta(D) \rightarrow 0} \sum_{\pi \in D}\|u(\pi)\|=L<+\infty .
$$

If $f(p, q), p=\left(p_{1}, p_{2}, p_{3}\right) \in K, q=\left(q_{1}, q_{2}, q_{3}\right) \in E_{3}$, is any function defined on $K \times E_{3}$ such that $f(p, t q)=t f(p, q)$ for all $t \geqq 0, p \in K, q \in E_{\mathbf{3}}$, let $U=\left[q \in E_{\mathbf{3}}\right.$, $\|q\|=1$ ] be the unit sphere in $E_{3}$, and suppose that $f(p, q)$ be bounded and uniformly continuous on $K \times U$. For every $\pi \in\{\pi\}$ let $\tau$ denote an arbitrary point $\tau \in \pi$, and let

$$
\Phi(\pi)=f[p(\tau), u(\pi)], \quad \pi \in\{\pi\} .
$$

By force of (6.i), $\Phi(\pi)$ is a quasi additive scalar function (with respect to $\mathfrak{D}$ and $\delta(D))$, and thus the following limit exists and is finite

$$
\begin{aligned}
\Im & =\int_{T} f(p, q)=\lim _{\delta(D) \rightarrow 0} \sum_{\boldsymbol{\pi} \in D} \Phi(\pi) \\
& =\lim _{\delta(D) \rightarrow 0} \sum_{\boldsymbol{x} \in D} f[p(\tau), u(\pi)],
\end{aligned}
$$

and this limit is independent of the choice of the points $\tau \in \pi, \pi \in D$. This limit is the integral of the function $f$ over the surface $T$ defined by the author [1, Appendix B]. This integral was used by J. M. Danskin, A. G. Sigalov, J. Cecconi, V. E. Bononcini, and the author in surface area theory and the calculus of variations. In [1, Appendix B] as well as in [9] it is shown that the same integral $\Im$ can be defined also by an analogous process with different classes $\{\pi\}$, functions $u$, and mesh $\delta$.

5. A lemma. If $\phi(I)=\left(\phi_{1}, \cdots, \phi_{k}\right), I \in\{I\}$, is any quasi additive vector function, let us denote by $\alpha_{r}=\alpha_{r}(I)$ the numbers $\alpha_{r}=\alpha_{r}(I)=\phi_{r}(I) /\|\phi(I)\|$, $r=1, \cdots, k$, provided $\|\phi(I)\|>0$. Thus $-1 \leqq \alpha_{r} \leqq 1$, and $\sum_{r} \alpha_{r}^{2}=1$. If $\|\phi(I)\|$ $=0$ let $\alpha_{r}=\alpha_{r}(I), r=1, \cdots, k$, be any set of $k$ real numbers with $\sum_{r} \alpha_{r}^{2}=1$. 
Thus $\alpha(I)=\left(\alpha_{1}, \cdots, \alpha_{k}\right), I \in\{I\}$, is a vector function defined for all $I \in\{I\}$, with $\|\alpha\|=1$.

(5.i) Let $\phi(I)=\left(\phi_{1}, \cdots, \phi_{k}\right), I \in\{I\}$, be any quasi additive function with $V=V(\|\phi\|)<+\infty$, let $\epsilon>0$ be an arbitrary number $0<\epsilon<(2 V+2)^{-1}$, let $\sigma=\epsilon^{3} / 48 k, \mu=\mu(\sigma), 0<\mu \leqq \sigma$, the number defined by (3.vii), and let $D_{0}=[I]$ be any system $D_{0} \in \mathfrak{D}$ with $\delta\left(D_{0}\right)<\mu$. Let $\lambda=\lambda\left(\sigma, D_{0}\right), 0<\lambda \leqq \mu$, be the further number defined by (3.vii), and let $D=[J]$ be any system $D \in \mathfrak{D}$ with $\delta(D)<\lambda$. For any $I \in D_{0}$ and $J \in D$ let $\alpha(I), \beta(J)$ be the unit vectors defined above. Let $\sum_{I}, \sum_{J}$, $\sum(I), \sum^{\prime}$ denote as usual sums ranging over all $I \in D_{0}$, all $J \in D$, all $J \in D$ with $J \subset I$, all $J \in D$ with $J \nsubseteq I$ for any $I \in D_{0}$ and let $\sum *$ be any sum ranging over all $J \in D$ with $J \subset I$ for some $I \in D_{0}$ and $\|\beta(J)-\alpha(I)\| \geqq \epsilon$. Then we have

$$
\begin{aligned}
& \sum^{*}\|\phi(J)\|<\epsilon, \quad \sum^{\prime}\|\phi(J)\|<\epsilon, \\
& \sum_{I} \sum(I)\|\phi(J)\|\|\alpha(I)-\beta(J)\|^{2}<\epsilon,
\end{aligned}
$$

and also

$$
\begin{gathered}
\sum_{I} \mid \sum^{(I)}\|\phi(J)\|-\|\phi(I)\|<\epsilon, \quad \sum_{I}\left\|\sum^{(I)} \phi(J)-\phi(I)\right\|<\epsilon, \\
\left\|\mathfrak{B}-\sum_{I} \phi(I)\right\|<\epsilon, \quad\left|V-\sum_{I}\|\phi(I)\|\right|<\epsilon, \\
\left\|\mathfrak{B}-\sum_{J} \phi(J)\right\|<\epsilon, \quad \mid V-\sum_{J}\|\phi(J)\|<\epsilon .
\end{gathered}
$$

Proof. By (3.vii) we have

$$
\begin{gathered}
\left\|\mathfrak{B}-\sum_{I} \phi(I)\right\|<\sigma, \quad \mid V-\sum_{I}\|\phi(I)\|<\sigma, \\
\left\|\mathfrak{B}-\sum_{J} \phi(J)\right\|<\sigma, \quad\left|V-\sum_{J}\|\phi(J)\|\right|<\sigma, \\
\sum_{I}\left\|\sum_{(I)}^{(I)} \phi(J)-\phi(I)\right\|<\sigma, \\
\sum_{I}\left|\sum^{(I)}\|\phi(J)\|-\|\phi(I)\|\right|<\sigma, \quad \sum^{\prime}\|\phi(J)\|<\sigma,
\end{gathered}
$$

and analogous relations hold for $\phi_{r}, \phi_{r}^{+}, \phi_{r}^{-}, r=1, \cdots, k$. For every $I \in D_{0}$ let $\sum{ }^{(* i)}$ denote any sum ranging over all $J \in D$ with $J \subset I,\|\alpha-\beta\| \geqq \epsilon$, where $\alpha=\alpha(I)=\left(\alpha_{1}, \cdots, \alpha_{k}\right), \beta=\beta(J)=\left(\beta_{1}, \cdots, \beta_{k}\right),\|\alpha\|=\|\beta\|=1$. We have

$$
\epsilon^{2} \leqq\|\alpha-\beta\|^{2}=\sum_{r}\left(\alpha_{r}-\beta_{r}\right)^{2}=2-2 \sum_{r} \alpha_{r} \beta_{r} .
$$

By multiplication by $\|\phi(J)\|$ we have, since $\beta_{r}=\phi_{r}(J) /\|\phi(J)\|$,

$$
\epsilon^{2}\|\phi(J)\| \leqq 2\left[\|\phi(J)\|-\sum_{r} \alpha_{r} \phi_{r}(J)\right]
$$

Let us observe that this bracket is $\geqq 0$ for every unit vector $\alpha$. Indeed, by the Schwarz inequality,

$$
\sum_{r} \alpha_{r} \phi_{r}(J) \leqq\left(\sum_{r} \alpha_{r}^{2}\right)^{1 / 2}\left(\sum_{r} \phi_{r}^{2}\right)^{1 / 2}=\|\phi(J)\| .
$$


By summation in (5.6) with respect to all $J \in D$ with $J \subset I,\|\alpha-\beta\| \geqq \epsilon$, we have

$$
\begin{aligned}
\epsilon^{2} \sum^{(* I)}\|\phi(J)\| & \leqq 2 \sum^{(* I)}\left[\|\phi(J)\|-\sum_{r} \alpha_{r} \phi_{r}(J)\right] \\
& \leqq 2 \sum^{(I)}\left[\|\phi(J)\|-\sum_{r} \alpha_{r} \phi_{r}(J)\right] \\
& =2\left[\sum^{(I)}\|\phi(J)\|-\sum_{r} \alpha_{r} \sum^{(I)} \phi_{r}(J)\right] .
\end{aligned}
$$

We shall denote as before by $u(I)=\left(u_{1}, \cdots, u_{k}\right)$ the vector $u(I)=\sum^{(I)} \phi(J)$ $-\phi(I)$, hence $u_{r}(I)=\sum \sum^{(I)} \phi_{r}(J)-\phi_{r}(I)$, and observe that by force of the fifth relation (5.5) we have $\sum_{I}\|u(I)\|<\sigma$. Thus by (5.7) we have

$$
\epsilon^{2} \sum^{(* I)}\|\phi(J)\| \leqq 2\left[\sum^{(I)}\|\phi(J)\|-\sum_{r} \alpha_{r}\left(\phi_{r}(I)+u_{r}(I)\right)\right],
$$

and since $\alpha_{r}=\phi_{r}(I) /\|\phi(I)\|$, by computation we deduce

$$
\epsilon^{2} \sum^{(* I)}\|\phi(J)\| \leqq 2\left[\sum^{(I)}\|\phi(J)\|-\|\phi(I)\|-\sum_{r} \alpha_{r} u_{r}(I)\right],
$$

where

$$
\left|\sum_{r} \alpha_{r} u_{r}\right| \leqq\left(\sum_{r} \alpha_{r}^{2}\right)^{1 / 2}\left(\sum_{r} u_{r}^{2}\right)^{1 / 2}=\left(\sum_{r} u_{r}^{2}\right)^{1 / 2}=\|u\|
$$

Thus

$$
\epsilon^{2} \sum^{(* I)}\|\phi(J)\| \leqq 2\left[\sum^{(I)}\|\phi(J)\|-\|\phi(I)\|\right]+2\|u(I)\|,
$$

and, by summation over all $I \in D_{0}$, also

$$
\begin{aligned}
\epsilon^{2} \sum^{*}\|\phi(J)\| & \leqq 2 \sum_{I}\left[\sum^{(I)}\|\phi(J)\|-\|\phi(I)\|\right]+2 \sum_{I}\|u(I)\| \\
& \leqq 2\left[\sum_{J}\|\phi(J)\|-\sum_{I}\|\phi(I)\|\right]+2 \sigma \\
& \leqq 2\left|V-\sum_{J}\|\phi(J)\|\right|+2\left|V-\sum_{I}\|\phi(I)\|\right|+2 \sigma,
\end{aligned}
$$

and, by the second and fourth relations (5.5), also

$$
\begin{aligned}
\epsilon^{2} \sum^{*}\|\phi(J)\| & \leqq 2 \sigma+2 \sigma+2 \sigma=6 \sigma \leqq \epsilon^{3} / 8 k<\epsilon^{3}, \\
\sum^{*}\|\phi(J)\| & <\epsilon / 8 k<\epsilon,
\end{aligned}
$$

and the first relation (5.1) is proved. The second relation (5.1) follows from the seventh relation (5.5) since $\sigma<\epsilon$. Note that we have $\|\alpha(I)-\beta(J)\|<\epsilon$ for all $J \in D, J \subset I$ except for those which appear in the sum $\sum^{(* I)}$, and for these we have obviously $\epsilon \leqq\|\alpha-\beta\|=\left[\sum_{r}\left(\alpha_{r}-\beta_{r}\right)^{2}\right]^{1 / 2} \leqq 2 k^{1 / 2}$. Hence

$$
\begin{aligned}
\sum_{I} & \sum(I)\|\phi(J)\|\|\alpha(I)-\beta(J)\|^{2} \\
& \leqq \epsilon^{2} \sum_{I} \sum^{(I)}\|\phi(J)\|+4 k \sum^{*}\|\phi(J)\| \\
& \leqq \epsilon^{2}\left(V+\epsilon^{3} / 48 k\right)+4 k[\epsilon / 8 k] \\
& \leqq \epsilon \cdot \epsilon(V+1)+\epsilon / 2 \leqq \epsilon / 2+\epsilon / 2=\epsilon,
\end{aligned}
$$

and (5.2) is proved. 
Relations (5.3), (5.4) are trivial consequences of the first six relations (5.5) since $\sigma<\epsilon$.

6. The concept of integral. Let $\phi(I)=\left(\phi_{1}, \cdots, \phi_{k}\right), I \in\{I\}$, be any quasi additive vector function (with respect to a system $\mathfrak{D}$ and a mesh function $\delta(D)$ as in $\$ \S 1,2)$. Suppose $V=V(\|\phi\|)<+\infty$. Then all functions $\phi_{r}$, $\|\phi\|, \phi_{r}, \phi_{r}^{+}, \phi_{r}^{-}, r=1, \cdots, k$, are quasi additive.

Let $T: p=p(w), w \in A, p=\left(x_{1}, \cdots, x_{m}\right)$, be any mapping from $A$ into a given set $K \subset E_{m}$. Then, for every $I \in\{I\}$, denote by $\omega(I)$ the oscillation of $T$ on $I$, i.e., the supremum of the differences $\|p(u)-p(v)\|$ for all $u, v \in I$. For every $D \in \mathfrak{D}, D=\{I\}$, let $\omega(D)=\max \omega(I)$ for all $I \in D$. Finally, let us suppose that the mesh function $\delta(D), D \in \mathcal{D}$, has been chosen in such a way that

$$
\omega(D) \leqq \delta(D)
$$

for all $D \in \mathfrak{D}$.

This is actually a continuity requirement for the mapping $T$ in $A$.

Let $p=\left(x_{1}, \cdots, x_{m}\right)$ denote any point $p \in E_{m}$ as above, $q=\left(q_{1}, \cdots, q_{k}\right)$ any point $q \in E_{k}$, and $\mathfrak{S}$ the unit sphere in $E_{k}$, or $\mathfrak{S}=\left[q \in E_{k},\|q\|=1\right]$. Let $f(p, q), p \in K \subset E_{m}, q \in E_{k}$, be any function of $(p, q)$ defined for all $(p, q)$ $\in K \times E_{k}$, such that

$\left(\mathrm{f}_{1}\right) f$ is a bounded and uniformly continuous function of $(p, q)$ in $K \times \mathfrak{S}$;

$\left(\mathrm{f}_{2}\right) f(p, t q)=t f(p, q)$ for all $t \geqq 0, p \in K, q \in E_{k}$.

Thus, by taking $f(p, 0)=0$ for every $p \in K$, the function $f(p, q),(p, q) \in K \times E_{k}$, is continuous in $K \times E_{k}$. Under hypothesis $(\mathrm{f})$ we shall understand $\left(\mathrm{f}_{1}\right)$ and $\left(\mathrm{f}_{2}\right)$.

For every $I \in\{I\}$ we may choose arbitrarily a point $\tau \in I$ and consider the set function

$$
\Phi(I)=f[p(\tau), \phi(I)], \quad I \in\{I\} .
$$

We shall prove the following main theorem:

(6.i) Theorem. If $\phi(I), I \in\{I\}$, is quasi additive (with respect to $D$ and $\delta(D)$ ) and $V=V(\|\phi\|)<+\infty$, if $(\omega)$ and $(\mathrm{f})$ hold, then $\Phi(I), I \in\{I\}$, is quasi additive (with respect to $\mathfrak{D}$ and $\delta(D)$ ), and, given $\epsilon>0$, the numbers $\eta(\epsilon), \lambda\left(\epsilon, D_{0}\right)$ of $\$ 2$ can be determined independently of the choice of $\tau$ in each set $I, I \in D$, $D \in \mathfrak{D}$.

The following statement is an immediate consequence of (6.i) and (2.v).

(6.ii) Theorem. If $\phi(I), I \in\{I\}$, is quasi additive and $V=V(\|\phi\|)<\infty$, if $(\omega)$ and $(\mathrm{f})$ hold, then the following limit exists and is finite:

$$
\Im=I(f, T, \phi)=\lim _{\delta(D) \rightarrow 0} \sum_{I \in D} f[p(\tau), \phi(I)],
$$

$-\infty<\Im<+\infty$, where $D=[I] \in \mathfrak{D}, \tau$ is any point $\tau \in I, I \in D, D \in \mathfrak{D}$, and $\Im$ is independent of the choice of $\tau$ on each $I \in D$.

The limit $\Im(f, T, \phi)$ is said to be the integral of the function $f$ over the 
mapping (or variety $T$ ) with respect to the quasi additive function $\phi$. We shall denote $\Im$ also by the notation

$$
\Im=\Im(f, T, \phi)=(A) \int f(p(w), \phi(I)) .
$$

We shall denote by $S(f, T, \phi, D)$ the sum under limit in (6.1).

Proof of (6.i). Since $f$ is bounded in $K \times \subseteq$, we have $|f(p, q)| \leqq N$ for some $N>0$ and all $p \in K, q \in \subseteq$. Let $V=V(\|\phi\|)$ and $M=\max [N, V+1]$. For every $I \in\{I\}$ we have defined in $\S 5$ the unit vector $\alpha(I)=\left(\alpha_{1}, \cdots, \alpha_{k}\right)$, and now, by requirement $\left(\mathrm{f}_{2}\right)$, we deduce

$$
\begin{aligned}
f[p(\tau), \phi(I)] & =f[p(\tau), \alpha(I)]\|\phi(I)\|, \\
|f[p(\tau), \phi(I)]| & \leqq M\|\phi(I)\|, \\
|S(T, f, \phi, D)| & \leqq M S(\|\phi\|, D) .
\end{aligned}
$$

If $V=0$ then we have also $\Im(f, T, \phi)=0$. We shall suppose $V>0$. Given $\epsilon>0$ then, by $\left(\mathrm{f}_{1}\right)$, there is a $\xi>0$ such that $\left|f(p, q)-f\left(p^{\prime}, q^{\prime}\right)\right|<\epsilon / 2 M$ for all $p, p^{\prime} \in K, q, q^{\prime} \in S,\left\|p-p^{\prime}\right\|<\xi,\left\|q-q^{\prime}\right\|<\xi$. We may suppose, furthermore, $\xi \leqq(2 V+2)^{-1}, \xi<1, \xi<\epsilon(6 M)^{-1}$. In harmony with (5.i), let $\sigma=\xi^{3} / 48 k$, $\mu=\mu(\sigma), 0 \leqq \mu \leqq \sigma$, the number defined by (3.vii), and let $D_{0}=[I]$ be any system $D_{0} \in \mathfrak{D}$ with $\delta\left(D_{0}\right)<\mu$. Let $\lambda=\lambda\left(\xi, D_{0}\right), 0<\lambda \leqq \mu$, the further number defined by (3.vii), and let $D=[J]$ be any system $D \in \mathfrak{D}$ with $\delta(D)<\lambda$. For any $I \in D_{0}$ and $J \in D$, let $\alpha(I), \beta(J)$ be the unit vectors defined in $\S 5$. Let $\sum_{I}, \sum_{J}, \sum^{(I)}, \sum^{\prime}$ be the sums defined as in (5.i), and $\sum^{*}$ any sum ranging over all $J \in D$ with $J \subset I$ for some $I \in D_{0}$ and $\|\beta(J)-\alpha(I)\| \geqq \xi$. Then we have

$$
\sum^{*}\|\phi(J)\|<\xi, \quad \sum^{\prime}\|\phi(J)\|<\xi .
$$

We shall also denote by $\sum^{(* I)}$ any sum ranging over all $J \in D$ with $J \subset I$ for some $I \in D_{0}$ and $\|\beta(J)-\alpha(I)\| \geqq \xi$, and by $\sum^{(0 I)}$ the complementary sum $\sum(0 I)=\sum(I)-\sum(* I)$. We have

$$
\left|V-\sum_{J}\|\phi(J)\|\right|<\xi, \quad \sum_{I}\left|\sum^{(I)}\|\phi(J)\|-\|\phi(I)\|\right|<\xi .
$$

We shall denote by $\tau, \tau^{\prime}$ arbitrary points $\tau \in I, \tau^{\prime} \in J$. Note that

$$
\begin{aligned}
& \sum^{*}\left|f\left[p\left(\tau^{\prime}\right), \phi(J)\right]\right| \leqq M \sum^{*}\|\phi(J)\| \leqq M \xi, \\
& \sum^{\prime}\left|f\left[p\left(\tau^{\prime}\right), \phi(J)\right]\right| \leqq M \sum^{\prime}\|\phi(J)\| \leqq M \xi<\epsilon .
\end{aligned}
$$

Note that for each $I \in D_{0}$ and all $J \subset I, J \in D$, the points $\tau^{\prime} \in J, \tau \in I$, lie in $I$, and by $\omega(D) \leqq \delta(D)$ we conclude that $\omega(I) \leqq \omega(D) \leqq \delta(D)<\lambda \leqq \mu \leqq \sigma<\xi$ and hence $\left\|p\left(\tau^{\prime}\right)-p(\tau)\right\| \leqq \omega(I)<\xi$. For the same $J, J \in D, J \subset I$, not included in the sum $\sum^{*}$ we have also $\|\beta(J)-\alpha(I)\|<\xi$, and hence

$$
\left|f\left[p\left(\tau^{\prime}\right), \beta(J)\right]-f[p(\tau), \alpha(J)]\right|<\epsilon / 2 M .
$$

Let 


$$
\Delta=\sum_{I}\left|\sum^{(I)} f\left[p\left(\tau^{\prime}\right), \phi(J)\right]-f[p(\tau), \phi(I)]\right|,
$$

and observe that $\sum^{(I)}=\sum^{(0 I)}+\sum^{(* I)}, \sum_{I} \sum^{(* I)}=\sum^{*}$. Hence

$$
\begin{aligned}
\Delta \leqq & \sum^{*}\left|f\left[p\left(\tau^{\prime}\right), \phi(J)\right]\right| \\
& +\sum_{I}\left|\sum^{(0 I)} f\left[p\left(\tau^{\prime}\right), \beta(J)\right]\|\phi(J)\|-f[p(\tau), \alpha(I)]\|\phi(I)\|\right| \\
\leqq & M \xi+\sum_{I} \mid \sum^{(0 I)}\left\{f\left[p\left(\tau^{\prime}\right), \beta(J)\right]-f[p(\tau), \alpha(I)]\right\}\|\phi(J)\| \\
& +f[p(\tau), \alpha(I)]\left\{\sum^{(0 I)}\|\phi(J)\|-\|\phi(I)\|\right\} \mid \\
\leqq & M \xi+(\epsilon / 2 M) \sum_{I} \sum^{(0 I)}\|\phi(J)\| \\
& +M \sum_{I}\left|\sum^{(0 I)}\|\phi(J)\|-\|\phi(I)\|\right| \\
\leqq & M \xi+(\epsilon / 2 M) \sum_{J}\|\phi(J)\|+M \sum_{I}\left|\sum^{(I)}\|\phi(J)\|-\|\phi(I)\|\right| \\
& +M \sum^{*}\|\phi(J)\| .
\end{aligned}
$$

By the relations (6.2) and (6.3) we have now

$$
\Delta \leqq M \xi+(\epsilon / 2 M)(V+\xi)+M \xi+M \xi \leqq 3 M \xi+\epsilon / 2<\epsilon / 2+\epsilon / 2=\epsilon .
$$

Relations (6.4) and (6.5) can be now rewritten in the form

$$
\sum_{I}\left|\sum^{(I)} \Phi(J)-\Phi(I)\right|<\epsilon, \quad \sum^{\prime}|\Phi(J)|<\epsilon,
$$

and this proves that $\Phi(I)$ is quasi additive, where $\delta\left(D_{0}\right)<\mu\left(\xi^{3} / 48 k\right)$ and $\delta(D)$ $<\lambda\left(\xi^{8} / 48 k, D_{0}\right)$, and the numbers $\mu, \lambda$ do not depend upon the choices of the points $\tau \in I, \tau^{\prime} \in J$. Thus (6.i) is proved.

\section{REFERENCES}

1. L. Cesari, Surface area, Princeton University Press, 1956.

2. S. Kempisty, Fonctions d'intervalle non additives, Actualités Sci. Ind. No. 824, Paris Hermann, 1939. 115-121.

3. H. Kober, On the existence of the Burkill integral, Canad. J. Math. vol. 10 (1957) pp.

4. G. de Rham, Variêtés différentiables, Actualités Sci. Ind. No. 1222, Paris, Hermann, 1955.

5. A. Rosenthal, Set functions, Albuquerque, University of New Mexico Press, 1948.

6. L. Tonelli, (a) Sulle funzioni di intervallo, Ann. Scuola Norm. Sup. Pisa (2) vol. 8 (1939) pp. 309-321; (b) Fondamenti di calcolo delle variazioni, 2 vols., Bologna, Zanichelli, 1922-1923.

7. H. Whitney, Geometric integration theory, Princeton University Press, 1957.

8. A. C. Zaanen, Theory of integration, New York, Interscience, 1958.

9. L. H. Turner, An invariant property of Cesari's surface integral, Proc. Amer. Math. Soc. vol. 9 (1958) pp. 920-925.

10. J. Serrin, $A$ new definition of the integral for non-parametric problems in the calculus of variations. Acta Math. vol. 102 (1959) pp. 23-32.

University OF Michigan, ANn Arbor, Michigan 